\section{7 - Sexualität und Akne}

40 - Sexualität und Psoriasis

42 - Sexualität und Fruchtbarkeit bei Autoimmundermatosen

\title{
Unansehliche Haut - große Probleme mit der Sexualität
}

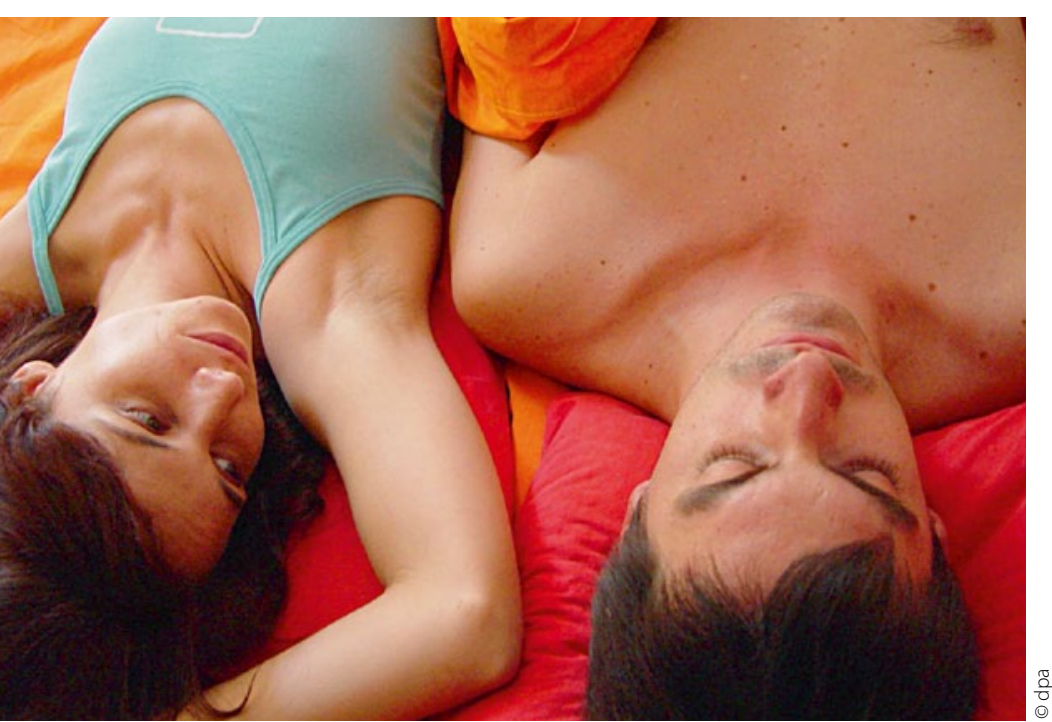

Für die Patienten ist es wichtig zu wissen, dass Sie ein offenes Ohr für ihre sexuellen Probleme haben.

— Die Haut als eines der größten Organe des Körpers grenzt das Individuum von seinen Mitmenschen ab. Daher ist es nicht verwunderlich, dass Hautveränderungen die Selbstsicherheit und Selbsteinschätzung der eigenen Attraktivität beeinträchtigen und damit auch die Partnerschaft und Sexualität stören können.

Aber einmal Hand auf das Herz! Wer fragt in der täglichen Routine nach, ob Patientinnen oder Patienten mit ausgedehnten, die sichtbaren Hautregionen oder den Genitalbereich betreffenden Hautveränderungen unter Einschränkungen ihres sexuellen Erlebens oder einer gestörten Kontaktaufnahme mit anderen Menschen leiden? Dabei müsste doch außer der Dermatologie eine Vielzahl von Fachdisziplinen (Innere Medizin, Allgemeinmedizin, Gynäkologie, Urologie, Kinder- und Jugendmedizin) mit solchen Problemen konfrontiert sein.
Der Beratungsbedarf betroffener Patienten ist vorhanden. In einer holländischen Studie äußerten sich nur 9\% der von Psoriasis betroffenen Patienten zufrieden mit der Aufmerksamkeit, die ihnen medizinisches Personal bezüglich sexueller Probleme entgegenbrachte (Meeuwis et al. Br J Dermatol 2011; 164: 1247). Die Ursachen dafür sind vielfältig. Das ärztliche Personal fühlt sich nicht ausreichend in sexualmedizinischen Themen ausgebildet; es besteht Sorge, dass solche Themen den zeitlichen (und finanziellen!) Rahmen der Sprechstunde sprengen könnten; teilweise ist das Thema auch von ärztlicher Seite aus mit Tabus beladen. Kann ich die ältere Patientin mit genitaler Psoriasis darauf ansprechen, ob sie sich in ihrer Sexualität beeinträchtigt fühlt? Dabei sollte es doch gerade Kolleginnen und Kollegen, die die Patienten und deren soziales Umfeld kennen, leichter fallen, das Thema anzusprechen!

Bei Befragungen von Allgemeinärzten in Norddeutschland gaben mehr als $70 \%$ an, dass „Sexualität nur selten ein Thema mit ihren männlichen Patienten" sei. Nur ein Drittel fühlte sich im Umgang mit Patienten, die über Sexualstörungen klagten, sicher. Das mag damit zusammenhängen, dass fast $90 \%$ der befragten Allgemeinärzte das Gefühl haben, im Studium nicht ausreichend über sexualmedizinische Störungsbilder informiert worden zu sein.

Aus diesen Fakten muss gefolgert werden, dass die Sensibilität der behandelnden Ärzte für sexualmedizinische Probleme bei Patienten mit Hauterkrankungen durch Aufklärung und Fortbildung erhöht werden muss. Zusätzlich wird aber erst die Vermittlung sexualmedizinischer Kompetenz im Studium und später durch Weiterbildungen die Versorgungslage entscheidend verbessern. Bezeichnend ist, dass bisher nur die Ärztekammer Berlin eine Zusatzweiterbildung in Sexualmedizin etabliert hat.

Ungeachtet dieser formalen Hemmnisse steht am Ende der Appell an die Kolleginnen und Kollegen, den Mut aufzubringen, sexualmedizinische Probleme anzusprechen. Für die Patienten ist es am Anfang entscheidend zu wissen, dass ihre behandelnden Ärzte überhaupt ein offenes Ohr für dieses Themengebiet haben. 